\section{Human gallbladder carcinoma: Role of neurotrophins, MIB-1, CD34 and CA15-3}

\author{
M. Artico, ${ }^{1}$ E. Bronzetti, ${ }^{2}$ \\ V. Alicino, ${ }^{1}$ B. lonta, \\ S. Bosco, ${ }^{3}$ C. Grande, ${ }^{3}$ \\ M. Bruno, ${ }^{1}$ F. M. Tranquilli Leali, ${ }^{2}$ \\ G. Ionta, ${ }^{4}$ L. Fumagalli ${ }^{2}$
}

'Department of Otorhinolaringology, Audiology and Phoniatry 'G. Ferreri'; 2Department of Human Anatomy; ${ }^{3}$ Department of Experimental Medicine, University of Rome 'La Sapienza'; ${ }^{4}$ Division of Oncological Surgery, ASL Scafati (SA), Rome, Italy

\section{Abstract}

Gallbladder carcinoma is the most common biliary tract tumor and the fifth most common gastrointestinal tract cancer. The prognosis of gallbladder carcinoma is poor and less than $5 \%$ of the patients are still alive five years postoperatively. Gallbladder specimens were obtained during surgical operations performed in eleven patients for resection of a gallbladder carcinoma, and during five autopsies (control cases selected among patients who died from for other causes, excluding those suffering from biliary or hepatic diseases). Immunohistochemical characterization and distribution of neurotrophins, with their respective receptors, were analyzed. The actual role played by these neurotrophic factors in the general regulation, vascular permeability, algic responsiveness, release of locally active substances and potential tumorigenesis in the gallbladder and biliary ducts compartment remains controversial. Our study revealed an increased immunohistochemical expression of NGF and TrKA in the epithelium and in the epithelial glands of the gallbladder carcinoma together with an evident immunoreactivity for BDNF in the same neoplastic areas. An evident immunoreactivity for NGF, TrKA and BDNF was observed in control specimens of gallbladder obtained during autopsies, whereas a weak or quite absent immunoreactivity was observed in the same specimens for NT4, TrKC and p75. On the contrary an appreciable immunoreactivity for p75 was observed in the specimens harvested from patients with gallbladder carcinoma. We also investigated the expression of some known tumor markers such as MIB-1 (anti Ki-67), CD34 and CA15-3, to identify a possible correlation between the expression of these molecular factors and the prognosis of gallbladder carcinoma. They resulted highly expressed in the stroma (CD34 and CA 153 ) and in the epithelium/epithelial glands (MIB-1) of the neoplastic areas and appeared to be almost absent in the control cases, suggesting that these markers, taken together, could be adopted as a panel of prognostic factors in the evaluation of the gallbladder carcinoma.

\section{Introduction}

Carcinoma of the gallbladder is the most common biliary tract tumor and the fifth most common gastrointestinal tract cancer. The symptoms of gallbladder carcinoma are nonspecific, but the risk is significantly higher in cholelithiasis. The non-specificity of symptoms is responsible for delayed diagnosis: in fact, this tumor is usually identified at an advanced stage when it has already become unresectable. ${ }^{1}$ The prognosis of gallbladder carcinoma is poor and less than $5 \%$ of the patients are still alive five years postoperatively. The majority of patients who live longer than five years are usually those in whom the carcinoma was first diagnosed at microscopic examination of their gallbladders which were removed for a presumed benign disease. ${ }^{1}$ The major route of spread of gallbladder cancer is locoregional: in fact, it usually extends directly into the liver and porta hepatis resulting in narrowing or obstruction of the common hepatic or right hepatic duct. ${ }^{1}$

The liver and the biliary ducts are innervated by both sympathetic and parasympathetic nerves, whose fibers are located around the hepatic artery, portal vein, intrahepatic and extrahepatic biliary ducts. ${ }^{2}$ Various neurotransmitters, as NPY, CGRP, VIP, somatostatin, enkephalin and bombesin, are involved in regulating the functions of the bile ducts, portal tract and arterial vessels of the hepatic compartment, with possible multiple effects on intrahepatic hemodynamics. ${ }^{3,4}$ Biliary ducts are the target of cholangiopathies, defined by a dysregulation of the equilibrium between cell growth and survival, with a progressive impairment of the proliferative response against duct injury leading to a functional liver failure at the end stage. ${ }^{2}$ The possible malignant transformation of cholangiocytes leads to cholangiocarcinoma, which may affect not only the biliary ducts, but also the gallbladder. These lesions, often diagnosed at late stages and with an increasing incidence worldwide, are characterized by a limited and unsatisfactory surgical option as well as by a minimal response to the conventional chemotherapy. ${ }^{5}$ We decided to investigate the localization and the distribution of
Correspondence: Marco Artico, Department of Otorhinolaringology, Audiology and Phoniatry 'G. Ferreri', University of Rome La Sapienza, V.le del Policlinico 155, 00161 Rome, Italy.

E-mail: marco.artico@uniromal.it

Key words: neurotrophins, human biliary ducts, gallbladder, immunohistochemistry, cholangiocarcinoma.

Received for publication: 25 September 2009. Accepted for publication: 7 January 2010.

This work is licensed under a Creative Commons Attribution 3.0 License (by-nc 3.0).

(C) Copyright M. Artico et al., 2010

Licensee PAGEPress, Italy

European Journal of Histochemistry 2010; 54:e10 doi:10.4081/ejh.2010.e10

neurotrophins and their receptors in this controversial area. Neurotrophins (NTs) constitute a family of dimeric proteins working as polypeptidic growth factors and acting like extracellular ligands. NTs, including NGF, BDNF, NT-3, NT-4, are involved in neuronal cell development, differentiation, survival and functional activities. Neurotrophins are also involved in the modulation of adult central nervous system functions and organization, as well as in the neural control of different activities related to vegetative innervation of several organs. ${ }^{6,7}$ Our previous observations regarding the localization and possible roles in different non-neuronal tissues such as those of the lymphoid, lung and prostate tissues $^{8,9}$ encouraged us to ascertain any possible involvement of NTs in the biliary ducts, both in physiological and pathological conditions (cholangiocarcinomas). Our study investigated the immunohistochemical localization and expression of NTs and their receptors both in normal autoptic specimens of normal gallbladder and in the neoplastic tissue obtained during surgical operations for the resection of a gallbladder carcinoma. Our interest was also focussed on some known tumor markers such as MIB-1 (Ki-67 antigen), CD34 and CA15-3 that are becoming significant in the diagnosis and evaluation of tumor but whose role in the gallbladder carcinoma has not yet been clearly established. ${ }^{10-12}$ We decided to describe the possibility of a correlation between the above mentioned tumor markers and the expression of NTs (and their receptors) to clarify the physiopathological mechanisms involved in gallbladder carcinoma development, and to identify a panel of reliable prognostic factors in the evaluation of tumor staging. 


\section{Materials and Methods}

\section{Patients}

Surgical operations were performed on eleven adult patients (age range 45-75 years) for the resection of a gallbladder carcinoma. Specimens harvested from the above mentioned cases, as well as five other specimens (control cases) obtained during autopsies or during surgical resections performed for gallbladder lithiasis, were then processed for immunohistochemical analysis. Experiments were performed in compliance with the Italian laws and guidelines concerning the patients informed consent. The following molecules were investigated: nerve growth factor (NGF), brain derived neurotrophic factor (BDNF), NT3 , NT-4 and the neurotrophin receptors tyrosine kinase A (TrKA), tyrosine kinase B (TrKB), tyrosine kinase $\mathrm{C}(\operatorname{TrKC})$ and $\mathrm{p} 75$, as well as MIB-1 (Ki-67 antigen), CD34 and CA15-3.

\section{Immunohistochemical analysis.}

Small fragments of gallbladder tissue were washed in PBS, fixed in 10\% formalin and embedded in paraffin according to a standard procedure. Serial $4 \mu \mathrm{m}$ thick sections were cut using a rotary microtome, mounted on gelatincoated slides and processed for immunohistochemistry. In order to study the immunolocalization of neurotrophins and their own receptors, the following antibodies were used: a) rabbit anti-nerve growth factor (anti-NGF) polyclonal antibody (Santa Cruz, CA, USA). It displayed $<1 \%$ cross-reactivity against recombinant human NT-3, NT-4 and BDNF; b) rabbit anti-tyrosine kinase A (anti-TrKA) polyclonal antibody (Santa Cruz). It recognized an epitope corresponding to amino acids 763 to 777 , mapping adjacent to the carboxy-terminus of human TrKA p140; c) goat polyclonal antibody to human p75 NT receptor (Santa Cruz). It recognized the amino acid sequence mapping the carboxy-terminus of the p75 NT receptor precursor of human origin; d) rabbit anti-brain derived neurotrophic factor (anti-BDNF) polyclonal antibody (Santa Cruz). It recognized the amino-terminal of mouse BDNF; e) rabbit antityrosine kinase B (anti-TrKB) polyclonal antibody (Santa Cruz). It recognized an epitope corresponding to amino acids 794 to 808 of mouse $\operatorname{TrKB}$ p145; f) rabbit anti-neurotrophin 3 (anti-NT-3) polyclonal antibody (Santa Cruz). It was raised against the amino-terminal of mouse NT-3; g) rabbit polyclonal antityrosine kinase C (anti-TrKC) antibody (Santa Cruz). It recognized an epitope corresponding to amino acids 798 to 812 of porcine TrKC p140; h) mouse monoclonal anti-human MIB-1 (Ki-67 antigen; Dako Cytomation, Denmark).
NORMAL
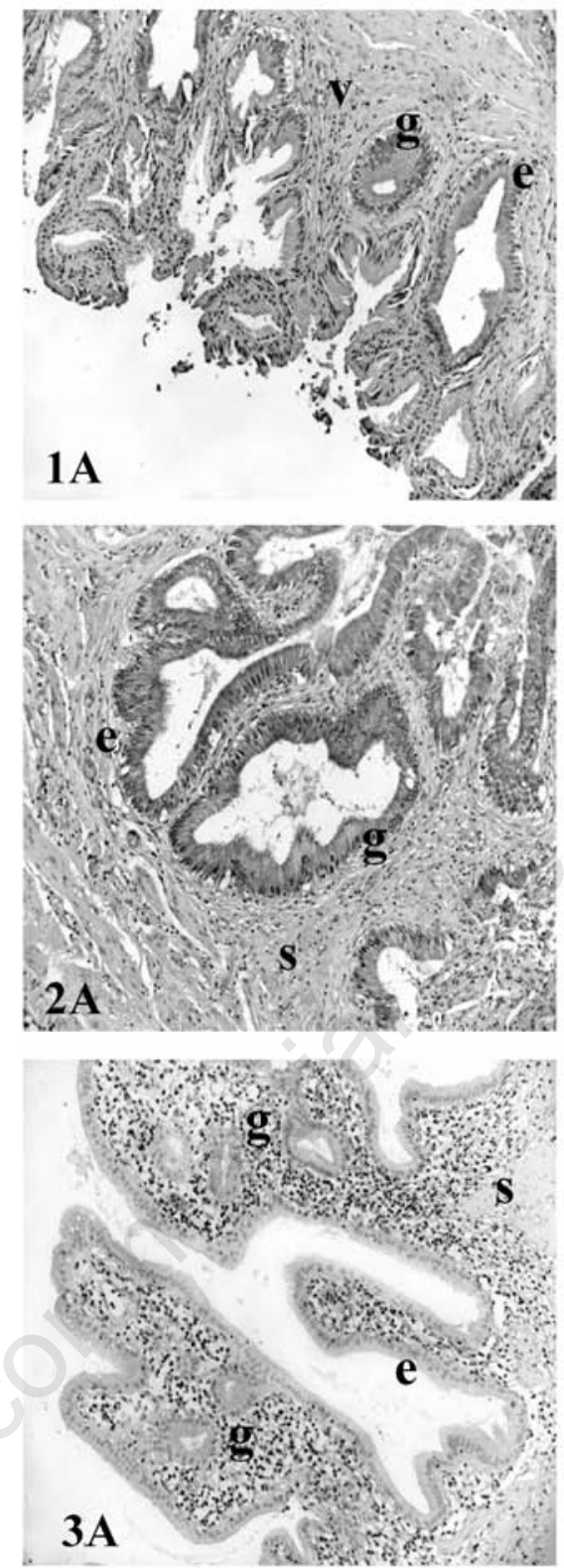

CARCINOMA
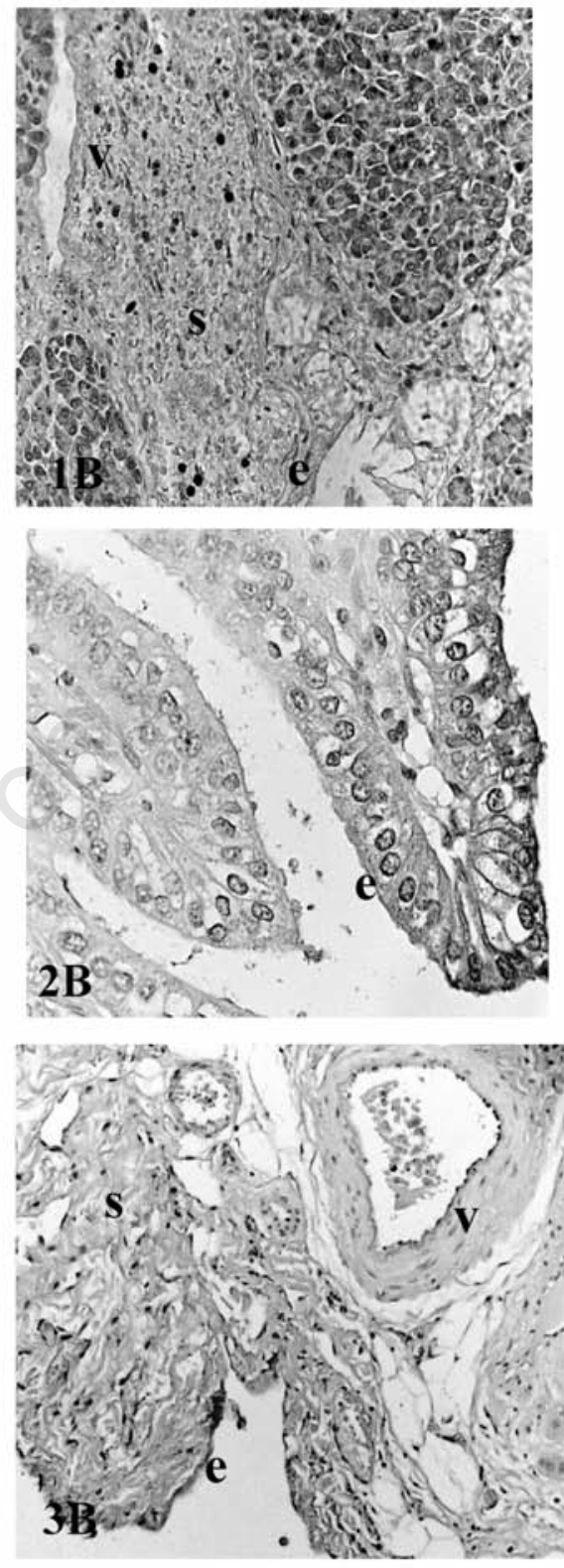

Figure 1. Micrographs of NGF immunostaining in human normal gallbladder (1A) and Gallbladder Carcinoma (1B). NGF shows an evident immunoreactivity in the epithelial glands (g) and in the blood vessels (v), weak in the epithelium (e), and in the normal gallbladder (1A). In gallbladder carcinoma NGF is highly detectable in the epithelium (e) and stroma (s), only moderate in in the blood vessels (v) (1B).

Figure 2. Micrographs of TrKA immunostaining in human normal gallbladder (2A) and Gallbladder Carcinoma (2B). In the normal gallbladder TrKA shows an evident reaction in the epithelium (e)/epithelial glands (g) but an absence in the blood vessels (v) and stroma (s) (2A). TrKA expression in gallbladder carcinoma is marked in the epithelium (e)/epithelial glands (g) but absent in blood vessels (v) and stroma (s) (2B).

Figure 3. Micrographs of p75 immunostaining in human normal gallbladder (3A) and Gallbladder Carcinoma (3B). P75 expression is generally absent in normal gallbladder (3A), evident in the epithelium (e) and stromal compartment (s), but only moderate in blood vessels (v) (3B).

The MIB-1 antibody recognizes native Ki-67 antigen and recombinant fragments of the Ki67 molecule, ${ }^{13}$ i) monoclonal mouse antihuman CA15-3 antibody (Dako Cytomation, Denmark) reacts with the DF-3 antigen, a high molecular weight $(300 \mathrm{kD})$ mucin-like glycoprotein, present on the apical borders of secretory mammary epithelial cells. The binding site for monoclonal antibody CA15-3 involves a glycosidic linkage between carbohydrate and pro- 
Table 1. Human normal gall bladder.

\begin{tabular}{|c|c|c|c|c|}
\hline & $\begin{array}{l}\text { Epithelium } \\
\text { glands }\end{array}$ & $\begin{array}{c}\text { Epithelial } \\
\text { vessels }\end{array}$ & Blood & Stroma \\
\hline NGF & \pm & + & $+/++$ & - \\
\hline TrKA & ++ & ++ & - & - \\
\hline P75 & - & - & - & - \\
\hline BDNF & ++ & ++ & - & + \\
\hline TrKB & \pm & - & - & + \\
\hline NT3 & ++ & ++ & ++ & ++ \\
\hline NT4 & - & - & - & - \\
\hline TrKC & \pm & \pm & - & \pm \\
\hline MIB & - & - & - & - \\
\hline CD34 & - & - & - & - \\
\hline CA15-3 & - & - & - & - \\
\hline
\end{tabular}

\pm , weak immunoreactivity; +, moderate immunoreactivity; ++ , relevant immunoreactivity, +++ , strong immunoreactivity.

Table 2. Gallbladder carcinoma.

\begin{tabular}{|c|c|c|c|c|}
\hline & $\begin{array}{l}\text { Epithelium } \\
\text { glands }\end{array}$ & $\begin{array}{l}\text { Epithelial } \\
\text { vessels }\end{array}$ & Blood & Stroma \\
\hline NGF & ++ & \pm & \pm & ++ \\
\hline TrKA & +++ & +++ & - & - \\
\hline P75 & ++ & - & + & ++ \\
\hline BDNF & +++ & + & \pm & +++ \\
\hline TrKB & - & - & - & $+/++$ \\
\hline NT3 & ++ & ++ & ++ & ++ \\
\hline NT4 & - & - & - & \pm \\
\hline TrKC & - & - & - & \pm \\
\hline MIB & ++ & +++ & \pm & + \\
\hline CD 34 & - & - & - & ++ \\
\hline CA15-3 & - & - & - & ++ \\
\hline
\end{tabular}

\pm , weak immunoreactivity; + , moderate immunoreactivity; ++ , relevant immunoreactivity, +++, strong immunoreactivity.

tein moieties on the DF3 antigen; l) mouse monoclonal anti-CD34 (Abcam plc, Cambridge, UK), which reacts with a protein present on endothelial cells and hematopoietic progenitor cells. The antibody recognizes a neuraminidase sensitive epitope on endothelium in vivo, particularly on small vessels and neoformed capillaries and developing vascular structures in embryonal structures. CD34 is highly expressed on hematopoietic progenitors, as well as on endothelial cells, brain, and testis. Staining for CD34 has been used to measure angiogenesis, which reportedly predicts tumor recurrence.

Incubation with primary antibodies was performed overnight at $4^{\circ} \mathrm{C}$ at a final concentration of $2-5 \mu \mathrm{g} / \mathrm{mL}$. Optimal antisera dilutions and incubation times were assessed in a series of preliminary experiments. After exposure to the primary antibodies, slides were rinsed twice in phosphate buffer and incubated 90 min at room temperature with the appropriate secondary antibody conjugated to horseradish

NORMAL
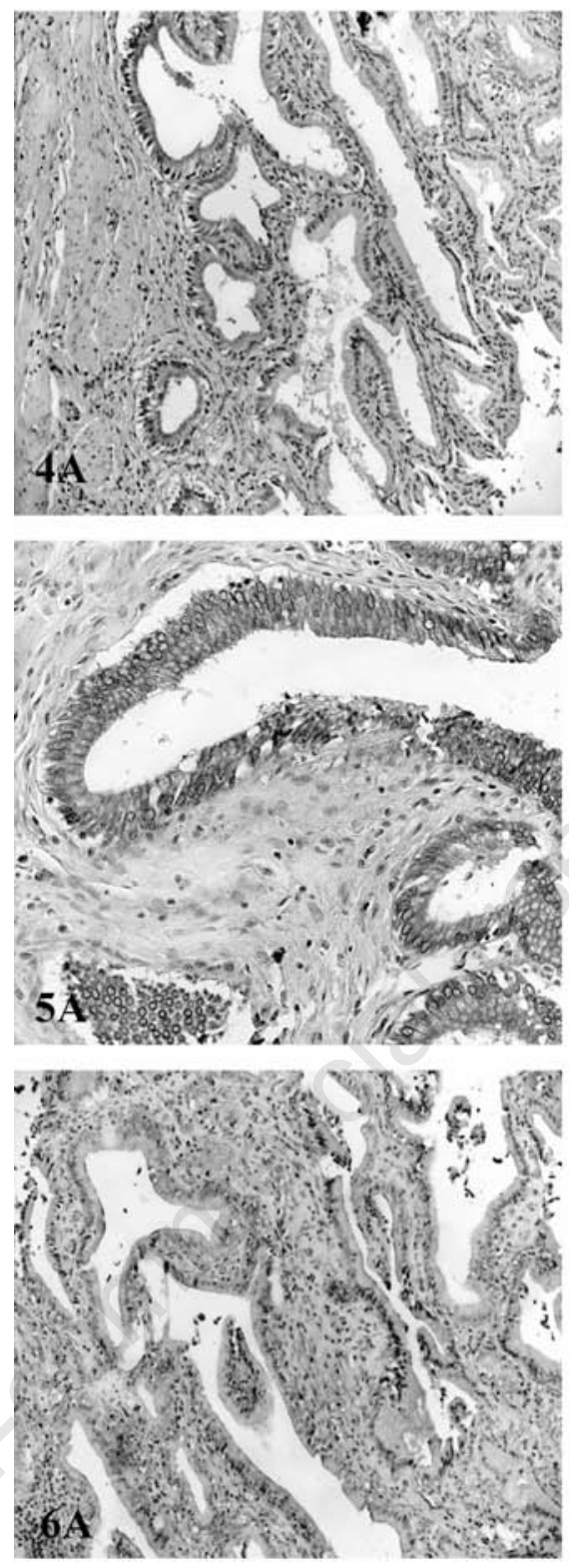

Figure 4 - 5. Micrographs of BDNF immunostaining in human normal gallbladder (4A$5 A)$ and Gallbladder Carcinoma $(4 B-5 B)$. BDNF expression is evident in epithelium (e)/epithelial glands $(\mathrm{g})$ and moderate in stromal compartment $(\mathrm{s})(4 \mathrm{~A}-5 \mathrm{~A})$. In gallbladder carcinoma BDNF shows a marked expression in the epithelium (e) and stroma (s), moderate in the epithelial glands $(\mathrm{g})$ and weak in blood vessels $(\mathrm{v})(4 \mathrm{~B}-5 \mathrm{~B})$.

Figure 6. Micrographs of TrKB immunostaining in human normal gallbladder (6A) and Gallbladder Carcinoma (6B). TrKB expression is moderate in the stroma (s) and weak in the epithelium (e), and in normal gallbladder $(6 \mathrm{~A})$, while it is evident in the stroma $(s)$ in gallbladder carcinoma (6B).

peroxidase (HRP) at final dilution 1:100. The secondary antibody-HRP linked against rabbit immunoglobulins was purchased from Boehringer (Mannheim GmbH, Mannheim, Germany), while secondary antibodies-HRP linked against mouse and goat immunoglobulins were from Sigma (Sigma Chemicals Co, St. Louis, M0, USA). After a further wash with phosphate buffer, slides were treated with 0.05\% 3,3-diaminobenzidine (DAB) and 0.1\% $\mathrm{H}_{2} \mathrm{O}_{2}$.

Finally, sections were counterstained with Mayer's hematoxylin and observed using a light microscope.

To block endogenous peroxidase activity, slides were pretreated with $3 \% \mathrm{H}_{2} \mathrm{O}_{2}$, whereas 
the non-specific binding of immunoglobulins was prevented by adding $3 \%$ fetal calf serum to the incubation medium. Negative control experiments were done: i) by omitting the primary antibody; ii) by substituting the primary antibody with an equivalent amount of nonspecific immunoglobulins; iii) by pre-incubating the primary antibody with the specific blocking peptide (antigen/antibody $=5$ according to supplier's instructions). The intensity of immune reaction was assessed microdensitometrically by an IAS 2000 image analyzer (Delta Sistemi, Rome, Italy) connected via a TV camera to the microscope. The system was calibrated taking the background obtained in sections exposed to non-immune serum as zero. Ten $100 \mu \mathrm{m}^{2}$ areas were delineated in each section by a measuring the diaphragm. Quantitative data of the intensity of the immune staining were analyzed statistically by analysis of variance (ANOVA) followed by Duncan's multiple range test as a post hoc test.

The parameter examined by quantitative analysis was the distribution and the intensity of the immunoreaction. The registered values were transformed into a single number expressed as conventional unit, including the standard deviation. This number can be read on the display of the Quantimet 500 image analyzer.

\section{Results}

\section{Immunohistochemistry}

Immunoreactivity for neurotrophins and their own receptors, as well as MIB-1, CD34 and CA15-3, was observed both in the normal gallbladder and in the neoplastic tissue and the results are described below (see Table I-II). The sections harvested from normal gallbladder generally revealed an evident immunoreactivity for NGF in the epithelial glands and in blood vessels (Figure 1A). TrkA immunoreactivity was strongly marked in the epithelial glands and in the epithelium layer (Figure 2A). A total absence of immunoreactivity for the p75NTR was demonstrated in all the examined structures (Figure 3A). BDNF immunoreactivity was extremely evident in the epithelial glands and in the epithelium (Figure 4A-5A) while in the same compartments we observed a weak reaction for TrkB (Figure 6A). Marked immunoreactivity was demonstrated for NT-3 in the epithelium, in the stroma, in the epithelial glands and in the blood vessels (Figure 7A). NT-4 in the previous compartments (Figure $8 \mathrm{~A}$ ) was characterized by the absence of immunoreactivty. TrkC immunoreactivity was poorly appreciable in the stroma and in epithelium/epithelial glands (Figure 9A). MIB1, CD34 and CA 15-3 immunoreactivity were
NORMAL
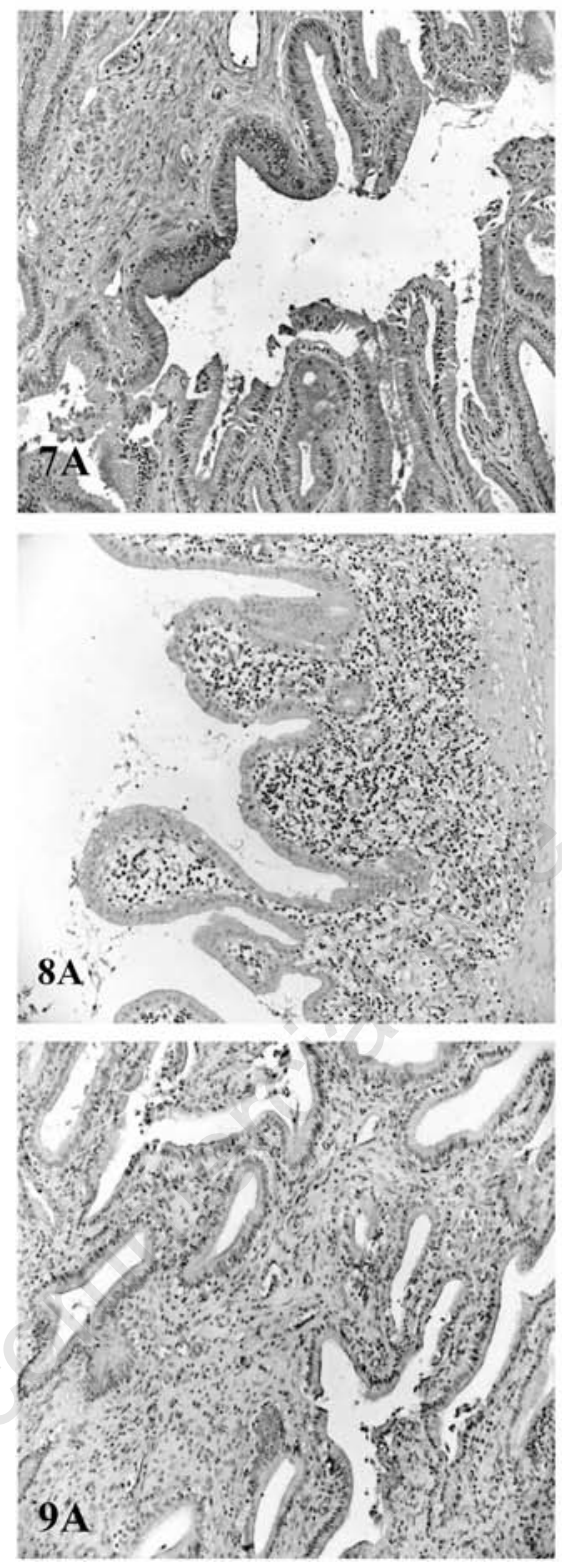

CARCINOMA
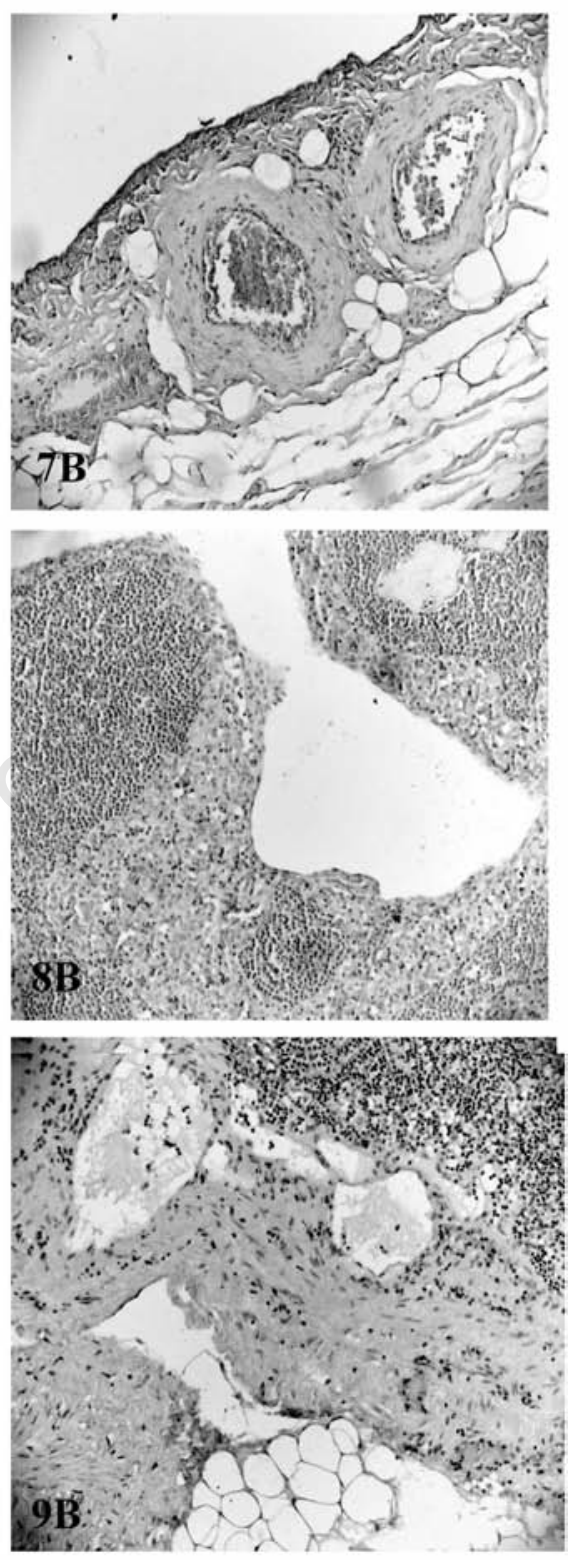

Figure 7. Micrographs of NT-3 immunostaining in human normal gallbladder (7A) and Gallbladder Carcinoma (7B). NT-3 is evident in all the compartments analysed (epithelium (e)/epithelial glands (g), blood vessels (v), stroma (s)) in normal gallbladder (7A), as well as in gallbladder carcinoma (7B).

Figure 8. Micrographs of NT-4 immunostaining in human normal gallbladder (8A) and Gallbladder Carcinoma (8B). NT-4 is generally absent in the normal gallbladder in the compartments described $(8 \mathrm{~A})$, but weak in the stroma $(s)$ in the gallbladder carcinoma $(8 B)$.

Figure 9. Micrographs of TrKC immunostaining in human normal gallbladder (9A) and Gallbladder Carcinoma (9B). TrKC is weakly expressed in the epithelium (e)/epithelial glands $(\mathrm{g})$ and stroma $(\mathrm{s})$ in normal gallbladder $(9 \mathrm{~A})$, but also weak only in the stroma $(\mathrm{s})$ of gallbladder carcinoma (9B).

generally absent in all the control specimens (figure not shown).

On the other hand, the gallbladder carcinoma samples revealed a relevant immunoreactivity for NGF in the epithelium and in the stroma, and an evident, to a lesser degree immunoreactivity, in the epithelial glands and in the blood vessels (Figure 1B). TrkA immuno-reactivity was strongly marked in all the compartments with the exception of blood vessels and stroma, with a maximal expression in the epithelium (Figure 2B). An evident 


\section{CARCINOMA}
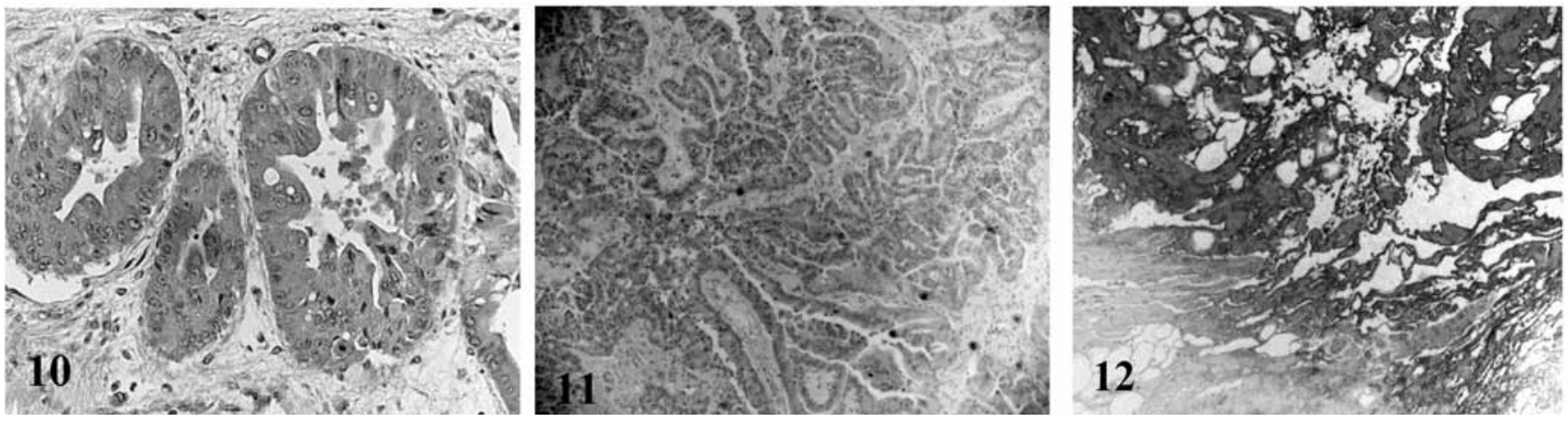

Figure 10. Micrographs of MIB-1 immunostaining in gallbladder carcinoma. MIB-1 is markedly expressed in the epithelial glands (g), appreciable in epithelium layer (e), moderate in the stroma (s) and weak in the blood vessels (v) of the neoplastic area.

Figure 11. Micrographs of CD34 immunostaining in gallbladder carcinoma. CD34 is clearly detectable only in the stromal compartment (s) of the neoplastic area.

Figure 12. Micrographs of CA15-3 immunostaining in gallbladder carcinoma. CA15- 3 is only clearly visible in the stromal compartment (s) of the neoplastic area.

immunoreactivity for the p75NTR was visible in the stroma and in the epithelium, but moderate in the blood vessels and absent in the epithelial glands (Figure 3B). BDNF immunoreactivity was extremely evident in the stroma and in the epithelium, moderate in the epithelial glands and poorly appreciable in the blood vessels (Figure 4B - 5B). A marked immunoreaction for TrkB was observed only in the stromal compartment (Figure 6B). Marked immunoreactivity was showed by NT-3 in the epithelium, stroma, epithelial glands and in the blood vessels (Figure 7B). NT-4 (Figure 8B) was characterized by the absence of immunoreactivity in the previous compartments with the exception for the stroma which appeared weakly positive (Figure 8B). TrkC immunoreactivity was only poorly appreciable in the stroma (Figure 9B). MIB-1 immunoreactivity was marked in the epithelium/epithelial glands, moderate in the stroma and very weak in the blood vessels (Figure 10). CD 34 (Figure 11) and CA15-3 (Figure 12) immunoreactivity was marked in the stroma and absent in the remaining compartments examined.

\section{Discussion}

NGF plays an inflammatory role, ${ }^{14}$ probably due to the direct action of NGF on mast cells and sensory neurones, as proposed by Woolf. ${ }^{15}$ Neurotrophins (NTs) are neurotrophic signalling polypeptides which include nerve growth factor (NGF), brain derived growth factor (BDNF), neurotrophin-3 (NT-3), NT-4/5 and NT-6, the latter apparently being specific to fish. ${ }^{16}$ The biological effects of NTs are mediated by the binding with two families of mem- brane receptors, the high affinity tyrosine kinase (TrK) and low affinity p75 receptor (p75NT receptor). ${ }^{6}$ TrKA is specifically activated by NGF, whereas TrKB and TrKC are primarily receptors for BDNF and NT-3 respectively. ${ }^{6}$ The physiological role of NTs in the development, maintenance and regeneration of the sympathetic and sensory nervous system has been well established ${ }^{6,16}$ and NGF mainly induces differentiation and decreases growth rate in a variety of neoplastic cells of neurogenic and non-neurogenic origin. ${ }^{17}$ Rasi et al. demonstrated that NGF was highly expressed not only in hepatocarcinoma (HCC) but also in early preneoplastic lesions, suggesting that NGF may have a role in the progression of HCC. ${ }^{18,19}$ We have investigated the possible role of NTs in the physiopathology of the gallbladder, especially in carcinoma specimens, together with the expression profile analysis of some tumor markers such as MIB-1 (anti Ki67), CD34 and CA15-3 whose role has been ascertained, but not clearly defined, in previous reports. ${ }^{10-12} \mathrm{Ki}-67$ expression appears to be a good diagnostic indicator for gallbladder carcinoma but not a prognostic factor for survival in patients with gallbladder carcinoma. ${ }^{10}$ Immunoreactivity for MIB-1 antigen is low in benign lesions such as chronic cholecystitis or hyperplasia when compared with gallbladder carcinoma, suggesting that epithelial hyperplasia with increased cellular proliferative activity plays an important role in carcinogenesis. In in situ gallbladder carcinoma MIB-1 expression was low compared with lesions showing more advanced gallbladder wall infiltration. ${ }^{20,21}$ Shrestha et al. found that patients with gallbladder carcinoma expressing high Ki-67 immunoreactivity had a worse postoperative prognosis than those who did not, although there was no relationship between
Ki-67 immunoreactivity and patient survival. ${ }^{20}$ Our results suggest a marked expression of its immunoreactivity within the epithelium/ epithelial glands of patients affected by gallbladder carcinoma, which partially concords with the results described by Hidalgo Grau $e t$ $a l .,^{10}$ confirming that epithelial hyperplasia with increased epithelial glands cellular proliferative activity plays a direct role in the tumorigenesis process. ${ }^{10}$ In fact, although MIB1 (Ki-67 antigen) cannot be individually assumed to be a reliable prognostic marker, the consistent immunoreaction level observed in the epithelial layer and in the epithelial glands of neoplastic specimens (compared to the total absence detected in the control cases) may be considered an important factor in the morphological differential diagnosis of gallbladder clinical features. CD34 antigen reveals only an appreciable immunoreactivity in the stromal compartment of neoplastic specimens, according to the work of Tanaka, ${ }^{12}$ which demonstrates that immunostaining for CD34 is negative, both in controls cases and in gallbladder cancer cells more specifically, since hematopoietic progenitor cells may differentiate into hepatic progenitor cells, hepatic progenitor cells can be identified by a hematopoietic stem cell marker, CD34, before they differentiate into hepatoblasts. ${ }^{12}$ Thus, we may confirm that CD34 is not a significant prognostic marker for evaluating the clinicopathological progression of gallbladder carcinoma.

Concerning the prognostic value of CA15-3, we may affirm that the sensitivity of this marker is more pronounced compared to MIB-1 and CD34. In fact, as Shukla's study, ${ }^{11}$ CA15-3 appears to be a reliable marker for discriminating gallbladder carcinomas from cholelithiasis cases, but it is less sensitive when used individually in gallbladder carcinoma. 
To conclude, we have demonstrated that in gallbladder carcinoma an increased expression of NGF, TrKA and BDNF is clearly visible, suggesting a direct role of these neurotrophic factors in the transformation and progression of neoplastic cells of the gallbladder tissue. We also investigated the expression of MIB-1, CD34 and CA 15 -3 and our results showed that, taken individually, they can be useful in the differential diagnosis of gallbladder clinical features, discriminating patients with primitive gallbladder carcinoma from those with cholelithiasis, but when considered as a whole, (especially MIB-1 and CA 15-3) they can represent a significant panel of prognostic factors, for evaluating the clinico-pathological progression of gallbladder carcinoma.

\section{References}

1. Adson MA. Carcinoma of the gallbladder. Surg Clin North Am 1973;53:1203.

2. Marzioni M. Nervous and neuroendocrine regulation of the pathophysiology of cholestasis and of biliary carcinogenesis. World J Gastroenterol 2006;12: 3471-80.

3. Lundberg JM, Terenius L, Hökfelt T et al. Neuropeptide Y (NPY)-like immunoreactivity in peripheral noradrenergic neurons and effects of NPY on sympathetic function. Acta Physiol Scand 1982;116:477-80.

4. Costa M, Furness JB. Somatostatin is present in a subpopulation of noradrenergic nerve fibres supplying the intestine. Neuroscience 1984;13:911-9.
5. Lazaridis KN. Cholangiocarcinoma. Gastroenterology 2005;128:1655-67.

6. Barbacid M. The TrK family of neurotrophin receptors. JNeuroimmunol 1994;25:1386-403.

7. Vega JA, Garcia-Suarez 0, Hannestad J, et al. Neurotrophins and the immune system. J Anat 2003;203:1-19.

8. Bronzetti E, Artico M, Pompili E, et al. Neurotrophins and meurotransmitters in human palatine tonsils: an immunohistochemical and RT-PCR analysis. Int J Mol Med 2006;18:49-58.

9. Bronzetti E, Artico M, Forte F, et al. A possible role of BDNF in prostate cancer detection. Oncol Rep 2008;19:969-74.

10. Hidalgo Grau LA, Badia JM, Admella Salvador C, et al. Gallbladder carcinoma: the role of p53 protein overexpression and Ki-67 antigen expression as prognostic markers. HBP (Oxford) 2004;6:174-80.

11. Shukla VK, Gurubachan, Sharma D, et al. Diagnostic value of serum CA242, CA 19-9, CA 15-3 and CA125 in patients with carcinoma of the gallbladder. Trop Gastroenterol 2006;27:160-5.

12. Tanaka S, Tanaka H, Yamamoto T, et al. Immunohistochemical demonstration of cKit protooncogene product in gallbladder cancer. J Hepatobil Pancreat Surg 2006;13:228-3.

13. Cattoretti G, Becker MHG, Key G, et al. Monoclonal antibodies against recombinants part of the Ki-67 antigen (MIB1 and MIB3) detect proliferating cells in microwave-processed formalin-fixed paraffin sections. J Pathol 1992;168:357-
63.

14. Stanisz AM, Stanisz JA. Nerve growth factor and neuroimmune interactions in inflammatory diseases. Ann NY Acad Sci 2000;917:268-72.

15. Woolf CJ, Ma QP. Peripheral cell types contributing to the hyperalgesic action of nerve growth factor in inflammation. $\mathrm{J}$ Neurosci 1996;16: 2716-23.

16. Snider WD. Functions of the neurotrophins during nervous system development: what the knockouts are teaching us. Cell 1994;77:627-38.

17. Kruttgen A, Schneider I, Weis J. The dark side of the NGF family: Neurotrophin in neoplasias. Brain Pathol 2006;16:304-10.

18. Rasi G, Serafino A, Bellis L, et al. Nerve growth factor involvement in liver cirrhosis and hepatocellular carcinoma. World $\mathrm{J}$ Gastroenterol 2007;13: 4986-95.

19. Oakley F, Trim N, Constandinou CM, et al. Hepatocytes express nerve growth factor during liver injury: evidence for paracrine regulation of hepatic stellate cell apoptosis. Am J Pathol 2003;163:1849-58.

20. Shrestha ML, Miyake H, Kikutsuji T, Tashiro S. Prognostic significance of Ki-67 and p53 antigen expression in carcinomas of bile duct and gallbladder. J Med Invest 1998;45:95-102.

21. Lee S. Differences in cell proliferation and prognostic significance of proliferating cell nuclear antigen and Ki-67 antigen immunoreactivity in situ and invasive carcinomas of the extrahepatic biliary tract. Cancer 1996;78:1881-7. 